\title{
A multicentre point prevalence study of delirium assessment and management in patients admitted to Australian and New Zealand intensive care units
}

Melissa J Ankravs, Andrew A Udy, Kathleen Byrne, Serena Knowles, Naomi Hammond, Manoj K Saxena, Michael C Reade, Michael Bailey, Rinaldo Bellomo and Adam M Deane, on behalf of the George Institute for Global Health and the Australian and New Zealand Intensive Care Society Clinical Trials Group www.doi.org/10.51893/2020.4.0a8

Published online first 7 December 2020

Delirium is independently associated with increased mortality, short and long term morbidity, and greater health care costs. ${ }^{1-3}$ The prevalence of delirium in patients admitted to an intensive care unit (ICU) varies widely, with reports of between $16 \%$ and $89 \%$ depending on how the diagnosis is made, the type of ICU and the geographical region studied. ${ }^{4-6}$

Australian and New Zealand data from a 2009 point prevalence study reported that routine delirium assessment occurred in only $3 \%$ of patients, with $9 \%$ of all patients identified as delirious and $7 \%$ of all patients requiring physical restraints. ${ }^{7}$ Since this study was published, international clinical practice guidelines have recommended that all critically ill adults should be regularly assessed for delirium using a validated tool. ${ }^{8}$ Moreover, preventing delirium and managing cognitive impairment is now a component of the Australian Commission on Safety and Quality in Health Care hospital accreditation.

There is limited evidence to guide treatment of delirious critically ill patients. ${ }^{9}$ Despite the lack of evidence to support the administration of drugs, international data suggest that antipsychotics are frequently administered; for example, in the United States, one in ten patients in the ICU is reported to receive an antipsychotic drug for either prevention or treatment of delirium. ${ }^{9,10}$ Use of physical restraint is controversial, with almost no evidence supporting or refuting its safety and effectiveness, and widespread variations in practice worldwide. ${ }^{8}$

Given the paucity of recent local data, changes to hospital accreditation, and international data suggesting widespread use of antipsychotic drugs in the critically ill, we collected data in the 2019 Australian and New Zealand Intensive Care Society Clinical Trials Group (ANZICS-CTG) Point Prevalence Program ${ }^{11}$ to provide up-to-date clinical practice evidence. We hypothesised that the majority of patients in the ICU would receive at least one formal assessment for delirium per day and the use of antipsychotic drugs and physical restraints would be limited to those patients with established hyperactive delirium.

\begin{abstract}
Objective: To characterise the assessment and management of delirium in patients admitted to intensive care units (ICUs) in Australia and New Zealand.
\end{abstract}

Methods: We conducted a multicentre observational point prevalence study across 44 adult Australian and New Zealand ICUs. Data were extracted for all patients in the ICU in terms of assessment and treatment of delirium. ICU-level data were collected regarding the use of explicit protocols related to delirium.

Results: We studied 627 patients, with 54\% (336/627) having at least one delirium screening assessment performed. The Confusion Assessment Method for the ICU (CAM-ICU) was the most frequently used tool $(88 \%$, 296/336). Of patients assessed, 20\% (68) were identified to have delirium. Eighteen per cent (111) of patients were administered a drug to manage delirium, with $41 \%$ (46) of those receiving a drug having no recorded assessment for delirium on that day. Of the drugs used to treat delirium, quetiapine was the most frequently administered. Physical restraints were applied to $8 \%(48 / 626)$ of patients, but only $17 \%(8 / 48)$ of such patients had been diagnosed with delirium. Most physically restrained patients either did not have delirium diagnosed $(31 \%, 15 / 48)$ or had no formal assessment recorded $(52 \%, 25 / 48)$ on that day.

Conclusions: On the study day, more than $50 \%$ of patients had a delirium screening assessment performed, with $20 \%$ of screened patients deemed to have delirium. Drugs that are prescribed to treat delirium and physical restraints were frequently used in the absence of delirium or the formal assessment for its presence.

Crit Care Resusc 2020; 22 (4): 355-360 


\section{ORIGINAL ARTICLES}

\section{Methods}

We conducted a multicentre observational point prevalence study across 44 Australian and New Zealand adult ICUs as part of the Point Prevalence Program coordinated by the George Institute for Global Health and ANZICS-CTG. ${ }^{11}$ Human research ethics committee or institutional approval for a waiver of individual patient consent was obtained for all participating sites.

The point prevalence data were recorded for all adult patients in a bed at participating ICUs at 10:00 hours on one of three assigned study days in June 2019. Study data were collected at each site and entered into an electronic data capture system (REDCap) ${ }^{12}$ hosted at the George Institute for Global Health. In addition to clinical and demographic information at ICU admission, data collected for the study day included whether the patient was assessed as delirious at any point during the study day, the type of delirium and the method of delirium assessment used for the first delirium status, the highest and lowest Richmond Agitation Sedation Scale (RASS) score recorded, administration of antipsychotic or specific $\alpha-2$ agonist drugs (dexmedetomidine or clonidine), and whether the patient was physically restrained at any time during the study day. Data collectors were not asked to speculate as to why any particular drug was administered. In addition, patient outcome data were reported at day 28 (counting the study day as day 1). Questions from the case report form are available in the Online Appendix. At an institutional level, sites also recorded if their ICU had a documented protocol, policy or guideline for the daily assessment of delirium in patients and, additionally, if their site had a document for delirium management.

\section{Statistical analysis}

Group comparison were performed using $\chi^{2}$ tests for equal proportion, Student $t$ test for normally distributed data, and Wilcoxon rank sum tests otherwise, with results reported as count (percentage), mean (standard deviation) and median (interquartile range) respectively. No imputation has been used for missing data, with missingness indicated using a denominator to designate availability. Analysis was conducted using SAS version 9.4 (SAS Institute, Cary, NC, USA), and a two-sided $P \leq 0.05$ was used to indicate statistical significance.

\section{Results}

A total of 44 ICUs in Australia $(82 \%, 36 / 44)$ and New Zealand $(18 \%, 8 / 44)$ participated in the study day. The majority of the sites were public hospitals, with private hospitals representing just $11 \%$ (5/44) of participating ICUs.

Data from 629 patients (Table 1) were collected as part of the Point Prevalence Program, with two patients excluded from our analysis due to lack of data in the delirium domain.

\section{Delirium assessment}

On the study day, 54\% (336/627) of patients had at least one screening assessment for delirium performed. Of these 336 patients, 32\% (109) had an assessment for delirium conducted once, $32 \%$ (108) of patients were assessed twice, and $35 \%$ (118) of patients had an assessment recorded three or more times on the study day. One patient was recorded as having an assessment for delirium conducted but the number of assessments was not provided.

The most frequent tool methodology to identify delirium was the Confusion Assessment Method for the ICU (CAMICU), with $88 \%(296 / 336)$ of the screened patients being evaluated using this method. "Clinical opinion" was the most commonly used method after CAM-ICU, with $6 \%$ (20/336) of patients assessed using this method. "Other" assessment was recorded for $5 \%$ (16/336) of patients. Four patients who had a delirium assessment had no response recorded as to the assessment method used. The Intensive Care Delirium Screening Checklist was not used to complete any assessments.

Of the patients who had at least one delirium assessment performed on the study day, delirium was present in $20 \%$ (68/336), representing $11 \%$ of all patients from the study day. For most patients diagnosed as delirious, the type of delirium was not specified, but when specified, hyperactive delirium was the most frequent manifestation recorded (Table 2).

\section{Sedation assessment}

During the study day, depth of sedation was quantified at least once in 89\% (556/627) of patients. Depth of sedation was categorised using the RASS for $80 \%$ (500/627), the Riker Sedation Agitation Scale for 8\% (50/627), the Ramsay Sedation Scale and "sedation assessed by other methods" for less than $1 \%$ each (4/627 and 2/627 respectively).

When limiting data to those patients who had the RASS performed, about one-quarter were at least deeply sedated or unarousable at one time point (Table 3). Forty-nine patients had a highest RASS score of -4 or less, with none of these patients having a CAM-ICU assessment performed.

\section{Drug administration}

During the study day, $18 \%(111 / 627)$ of patients were administered at least one drug commonly prescribed to treat delirium, with $23 \%$ (25/111) of these patients receiving more than one of the listed drugs. Of the 111 patients who received at least one of these drugs, $41 \%$ (46) did not have any assessment for delirium recorded on the study day.

Of the drugs used to treat delirium, quetiapine was the most frequently administered (Table 4) and 12\% (74/627) of all patients on the study day received an antipsychotic drug. On review of these 74 patients who received at least one 


\section{ORIGINAL ARTICLES}

Table 1. Characteristics of study patients

\begin{tabular}{|c|c|c|c|c|}
\hline Characteristics & All patients & Delirium & $\begin{array}{l}\text { No delirium } \\
\text { recorded* }\end{array}$ & $P$ \\
\hline Total number of patients & 627 & 68 & 559 & \\
\hline Sex, female & $250(39.9 \%)$ & $21(30.9 \%)$ & $229(41 \%)$ & 0.11 \\
\hline Weight $(\mathrm{kg})$, mean $(\mathrm{SD})^{\dagger}$ & $82.3 \pm 23.2$ & $83.3 \pm 23.2$ & $82.2 \pm 23.2$ & 0.72 \\
\hline Age (years), median (IQR) & $62(48-72)$ & $67(50.5-75.5)$ & $62(48-71)$ & 0.05 \\
\hline APACHE II, median (IQR) $)^{\ddagger}$ & $16(12-21)$ & $17(13-22)$ & $16(12-21)$ & 0.09 \\
\hline Days from ICU admission to study day, median (IQR) & $3(1-7)$ & $4(1.5-8.5)$ & $3(1-6)$ & 0.05 \\
\hline Source of ICU admission & & & & 0.60 \\
\hline Emergency department & $199(31.6 \%)$ & $20(29.4 \%)$ & $179(31.9 \%)$ & \\
\hline Operating theatre after elective surgery & $131(20.8 \%)$ & $15(22.1 \%)$ & $116(20.7 \%)$ & \\
\hline Ward & $126(20 \%)$ & $11(16.2 \%)$ & $115(20.5 \%)$ & \\
\hline Operating theatre after emergency surgery & $93(14.8 \%)$ & $12(17.6 \%)$ & $81(14.4 \%)$ & \\
\hline Other ICU & $39(6.2 \%)$ & $3(4.4 \%)$ & $36(6.4 \%)$ & \\
\hline Other hospital (except from other ICU) & $39(6.2 \%)$ & $7(10.3 \%)$ & $32(5.7 \%)$ & \\
\hline Previous ICU admission in this hospital admission & $42(6.7 \%)$ & $5(7.4 \%)$ & $37(6.6 \%)$ & 0.82 \\
\hline Invasive mechanical ventilation ${ }^{\ddagger \S}$ & $286(45.7 \%)$ & $32(47.1 \%)$ & $254(45.5 \%)$ & 0.81 \\
\hline Respiratory support" & 165 (26.3\%) & $22(32.4 \%)$ & $143(25.6 \%)$ & 0.23 \\
\hline
\end{tabular}

APACHE = Acute Physiological and Chronic Health Evaluation; ICU = intensive care unit; IQR = interquartile range; SD = standard deviation. * Includes patients who have been screened as not delirious and those not screened. $+n=614$. $\neq n=626$. § At any point during the study day. I Using supplemental high flow nasal prongs, continuous positive airway pressure or bi-level airway pressure at any point during the study day.

antipsychotic drug, 36\% (27) did not have any assessment for delirium recorded on the study day.

For the patients deemed to have hyperactive or mixed hyperactive/hypoactive delirium, $85 \%(17 / 20)$ received a listed antipsychotic or $\alpha-2$ agonist drug. For patients deemed to have hypoactive delirium, 30\% (3/10) received a listed drug.

\section{Physical restraints}

Data on the presence of a physical restraint were recorded for 626 patients, with $8 \%(48 / 626)$ of patients being restrained at some time point during the study day. Of the patients physically restrained, only $17 \%(8 / 48)$ were diagnosed with delirium, with $31 \%(15 / 48)$ screened as not having delirium and $52 \%$ (25/48) having no formal assessment recorded. Of the 8 patients who were physically restrained and diagnosed with delirium, 63\% (5/8) were considered to have either hyperactive delirium or a combination of both hyperactive and hypoactive delirium. Of those restrained, 35\% (17/48) also had a drug administered likely to have been used to treat delirium.

\section{Patient outcomes}

At day $28,23 \%(143 / 627)$ of patients had not yet been discharged from ICU or hospital. For those patients who had been discharged, 83\% (403/484) of patients were alive at hospital discharge.

\section{Unit level data}

For the 44 ICUs participating in the study, 77\% (34/44) had a documented protocol, policy or guideline for the daily assessment of delirium, and 70\% (31/44) had a documented protocol, policy or guideline for the management of delirium.

For the 336 patients assessed for the presence of delirium on the study day, $88 \%$ (297/336) were admitted to an ICU that had a delirium assessment protocol. For the 111 patients who received at least one drug commonly prescribed to treat delirium, 79\% (88/111) were admitted to an ICU that had a delirium management protocol.

\section{Discussion}

\section{Key findings}

We observed that just over half of ICU patients on the study day received at least one formal assessment for delirium. Furthermore, we observed that drugs commonly used to treat delirium (antipsychotic and $\alpha-2$ agonists) and physical restraints were widely administered but were not limited 


\section{ORIGINAL ARTICLES}

Table 2. Presence of delirium from the first delirium assessment recorded on the study day

\begin{tabular}{lc} 
Type & Number (\%)* \\
\hline Total number of patients & 336 \\
\hline Not delirious & $266(79 \%)$ \\
Type of delirium not specified & $38(11 \%)$ \\
Hyperactive delirium & $18(5 \%)$ \\
Hypoactive delirium & $10(3 \%)$ \\
Mixed hyperactive/hypoactive delirium & $2(0.6 \%)$ \\
\hline
\end{tabular}

* Number of patients who had delirium status assessed at least once. The numbers do not add up to 336 patients as two patients who had their delirium status assessed did not have a type recorded.

to those patients with established hyperactive delirium. In particular, more than one-third of patients who received antipsychotic drugs such as quetiapine, olanzapine and haloperidol did not have a delirium assessment recorded and, of the patients physically restrained, less than a fifth were diagnosed with delirium and more than half had no formal delirium assessment documented on that day.

\section{Comparison with previous studies}

In the 10 years between the current and previous point prevalence studies, routine delirium assessment in Australian and New Zealand ICUs has increased from very infrequent to about half of all patients being assessed. Moreover, there appears to be a marked change in the preferred assessment tool. The initial validation studies for the CAM-ICU were published in 2001, ${ }^{13,14}$ but CAM-ICU was not used in any patients in 2009, whereas it is now the most frequently used method of assessment. During this period, many Australian and New Zealand ICUs have been involved in trials endorsed by ANZICS-CTG which included delirium assessments using CAM-ICU, ${ }^{15,16}$ suggesting that site participation in well conducted trials may contribute to changes in work practices after trial completion. The prevalence of delirium in patients assessed appears to have increased from 9\% in 2009 to $20 \%$ in this study. This may reflect a greater awareness of the process and increased diagnosis or a true increase in prevalence. While more frequently diagnosed, it is uncertain whether there has been any variation in treatment: the use of physical restraints was similar between 2009 (7\%) and $2019(8 \%)$, but drugs that treat delirium were not recorded in the 2009 dataset.

A retrospective observational cohort study across 71 ICUs in the United States in 2010 reported antipsychotics were administered to $11 \%$ of patients, with haloperidol being the most frequently administered antipsychotic. ${ }^{10}$ Our data suggest similarities between our region and the United States, with $12 \%$ of patients in Australia and New Zealand receiving at least one antipsychotic on the study day, with quetiapine being the most commonly used antipsychotic and $\alpha-2$ agonists also frequently administered. Local prescribing practice suggests that clinicians are more likely to prescribe drugs that have been reported as superior to placebo in randomised clinical trials. 1,15-17

We also observed that, in Australian and New Zealand ICUs, hypoactive delirium is infrequently documented (3\% screened) and, when present, is not usually treated with an antipsychotic drug or $\alpha-2$ agonist. This suggests some differences between our region and the United States, as a large well conducted multicentre trial evaluating antipsychotic drugs in 566 patients with diagnosed delirium reported hypoactive delirium as the predominant syndrome — present in almost $90 \%$ of patients with delirium. ${ }^{1}$

\section{Clinical implications}

Despite $77 \%$ (34/44) of sites having a documented protocol, policy or guideline for the daily assessment of delirium in the ICU, just over half of the patients included had a delirium assessment completed on the study day. Moreover, $70 \%$ (31/44) of sites had a document policy for the management of delirium in the ICU, but antipsychotic drugs, $\alpha-2$ agonists and physical restraints were frequently

\section{Table 3. Sedation category according to Richmond Agitation Sedation Scale (RASS) for patients who had a highest}

and lowest RASS score recorded on the study day

\begin{tabular}{|c|c|c|}
\hline RASS score & Description & $\begin{array}{c}\text { Patients with a RASS score } \\
\text { recorded }\end{array}$ \\
\hline Lowest RASS score $\geq 1$ & $\begin{array}{l}\text { Restless through to overtly combative every time RASS } \\
\text { recorded on the study day }\end{array}$ & $2 \%(9)$ \\
\hline Highest RASS score $\geq 1$ & $\begin{array}{l}\text { Restless through to overtly combative for at least part of the } \\
\text { study day }\end{array}$ & $25 \%(126)$ \\
\hline Highest RASS score $\leq-4$ & $\begin{array}{l}\text { Deep sedation/unarousable every time RASS recorded on the } \\
\text { study day }\end{array}$ & $10 \%(49)$ \\
\hline Lowest RASS score $\leq-4$ & Deep sedation/unarousable for at least part of the study day & $23 \%(115)$ \\
\hline
\end{tabular}




\section{ORIGINAL ARTICLES}

\begin{tabular}{|c|c|c|}
\hline \multicolumn{3}{|c|}{$\begin{array}{l}\text { Table 4. Administration of drug (via any route) that } \\
\text { may be used to treat delirium }\end{array}$} \\
\hline Drug & $\begin{array}{l}\text { Patients who } \\
\text { received } \\
\text { drug* }\end{array}$ & $\begin{array}{l}\text { Patients who } \\
\text { received drug and } \\
\text { had a delirium } \\
\text { assessment } \\
\text { completed* }\end{array}$ \\
\hline Quetiapine & 35 & 21 \\
\hline Clonidine & 31 & 17 \\
\hline Dexmedetomidine & 30 & 18 \\
\hline Olanzapine & 27 & 17 \\
\hline Haloperidol & 18 & 11 \\
\hline Risperidone & 4 & 4 \\
\hline \multicolumn{3}{|c|}{$\begin{array}{l}\text { * The total number of patients does not equal the } 111 \text { patients who } \\
\text { definitely received a drug, as some patients received more than one } \\
\text { listed drug on the study day. }\end{array}$} \\
\hline
\end{tabular}

administered to patients without documented hyperactive delirium. Given the adverse effects of these therapies and the fluctuant nature of delirium, ${ }^{18}$ we believe that this observation should encourage local quality assurance programs to ensure that the use of these interventions is limited to only those patients with confirmed delirium.

\section{Strengths and limitations}

A major strength of our study is that we were able to provide a snapshot of current delirium assessment and management practices across a large number of Australian and New Zealand ICUs.

Limitations include that we may have missed assessments of delirium on the study day, which were recorded in a way that local data collectors did not recognise or could not ascertain. ${ }^{5}$ Moreover, patients may have had prominent hyperactive delirium before the study day, and drugs may have been initiated before the study day and a decision made to discontinue ongoing delirium assessment. In addition, the indications for drugs and physical restraints were not recorded, and it is possible that some of the drugs were prescribed for an indication unrelated to delirium (eg, clonidine prescribed for the management of hypertension) or just a continuation of a regularly prescribed drug. However, given the impact of chemical and/or mechanical restraints on patients, we suggest that an assessment for delirium should occur at least once each day that these therapies are applied. ${ }^{8}$ Furthermore, we did not record the total daily dose of any drug or how often the drug was administered on the study day. These indices of treatment intensity and likely risk of adverse effects would be important to assess in a more focused single-centre quality improvement initiative.

\section{Future directions}

The use of restraints in a health care setting has psychological, ethical and legal ramifications, ${ }^{19}$ but unrestrained hyperactive delirious patients may cause harm to themselves or staff. ${ }^{20}$ Thus, it appears prudent that future research includes evaluation as to the effects of chemical and physical restraints on patient-centred outcomes and both patient and health care worker safety.

Due to its prevalence, our data also suggest that future trials of delirium treatment in Australia and New Zealand should focus on hyperactive delirium and the administration of quetiapine, $\alpha-2$ agonists and olanzapine, as these are currently the most frequently administered drugs.

\section{Conclusion}

Our study reports that more than half of patients in Australian and New Zealand ICUs have at least a single daily delirium assessment. Delirium was identified in about $20 \%$ of patients who had a delirium assessment. Despite the majority of sites having a protocol, policy or guideline for delirium assessment and management, our data suggest that drug therapy and physical restraints may be administered outside of local recommendations.

Acknowledgements: We thank all the contributing sites (Online Appendix), research nurses, and coordinators, the George Institute for Global Health and the Australian and New Zealand Intensive Care Society Clinical Trials Group Point Prevalence Program. The Royal Melbourne Hospital Intensive Care Academic Fund kindly provided financial support for this study.

\section{Institution where work was performed}

Department of Intensive Care, Royal Melbourne Hospital, Melbourne, VIC, Australia.

\section{Competing interests}

None declared.

Author details
Melissa J Ankravs
Andrew A Udy
Kathleen Byrne
Serena Knowles
Naomi Hammond
Manoj K Saxena
Michael C Reade
Michael Bailey
Rinaldo Bellomo
Adam M Deane
Ad,2




\section{ORIGINAL ARTICLES}

On behalf of the George Institute for Global Health and the Australian and New Zealand Intensive Care Society Clinical Trials Group

1 The University of Melbourne, Melbourne Medical School, Department of Medicine and Radiology, Royal Melbourne Hospital, Melbourne, VIC, Australia.

2 Intensive Care Unit, Royal Melbourne Hospital, Melbourne, VIC, Australia.

3 Pharmacy Department, Royal Melbourne Hospital, Melbourne, VIC, Australia.

4 Intensive Care Unit, The Alfred Hospital, Melbourne, VIC, Australia.

5 Australian and New Zealand Intensive Care Research Centre, School of Public Health and Preventative Medicine, Monash University, Melbourne, VIC, Australia.

6 Critical Care Division, The George Institute for Global Health, University of New South Wales, Sydney, NSW, Australia.

7 Malcolm Fisher Department of Intensive Care Medicine, Royal North Shore Hospital, Sydney, NSW, Australia.

8 Intensive Care Unit, Bankstown Hospital, Sydney, NSW, Australia.

9 Royal Brisbane and Women's Hospital Clinical School, Faculty of Medicine, University of Queensland, Brisbane, QLD, Australia.

10 Joint Health Command, Australian Defence Force, Canberra, $\mathrm{ACT}$, Australia.

11 Intensive Care Unit, Austin Hospital, Melbourne, VIC, Australia.

Correspondence: melissa.ankravs@mh.org.au

\section{References}

1 Girard TD, Exline MC, Carson SS, et al. Haloperidol and ziprasidone for treatment of delirium in critical illness. N Engl J Med 2018; 379: 2506-16.

2 Mitchell ML, Shum DHK, Mihala G, et al. Long-term cognitive impairment and delirium in intensive care: A prospective cohort study. Aust Crit Care 2018; 31: 204-11.

3 Girard TD, Thompson JL, Pandharipande PP, et al. Clinical phenotypes of delirium during critical illness and severity of subsequent long-term cognitive impairment: a prospective cohort study. Lancet Respir Med 2018; 6: 213-22.

4 Reade MC, Finfer S. Sedation and delirium in the intensive care unit. N Engl J Med 2014; 370: 444-54.

5 Holmes NE, Amjad S, Young M, et al. Using language descriptors to recognise delirium: a survey of clinicians and medical coders to identify delirium-suggestive words. Crit Care Resusc 2019; 21: 299-302.

6 Patel MB, Bednarik J, Lee $P$, et al. Delirium monitoring in neurocritically ill patients: a systematic review. Crit Care Med 2018; 46: 1832-41.

7 Elliott D, Aitken LM, Bucknall TK, et al. Patient comfort in the intensive care unit: a multicentre, binational point prevalence study of analgesia, sedation and delirium management. Crit Care Resusc 2013; 15: 213-9.

8 Devlin JW, Skrobik Y, Gelinas C, et al. Clinical practice guidelines for the prevention and management of pain, agitation/sedation, delirium, immobility, and sleep disruption in adult patients in the ICU. Crit Care Med 2018; 46: e825-73.

9 Burry L, Hutton B, Williamson DR, et al. Pharmacological interventions for the treatment of delirium in critically ill adults. Cochrane Database Syst Rev 2019; (9): CD011749.

10 Swan JT, Fitousis K, Hall JB, et al. Antipsychotic use and diagnosis of delirium in the intensive care unit. Crit Care 2012; 16: R84.

11 Thompson K, Hammond N, Eastwood G, et al. The Australian and New Zealand Intensive Care Society Clinical Trials Group Point Prevalence Program, 2009-2016. Crit Care Resusc 2017; 19: 88-93.

12 Harris PA, Taylor R, Thielke R, et al. Research electronic data capture (REDCAp) - a metadata-driven methodology and workflow process for providing translational research informatics support. J Biomed Inform 2009; 42: 377-81.

13 Ely EW, Margolin R, Francis J, et al. Evaluation of delirium in critically ill patients: validation of the Confusion Assessment Method for the Intensive Care Unit (CAM-ICU). Crit Care Med 2001; 29: 1370-9.

14 Ely EW, Inouye SK, Bernard GR, et al. Delirium in mechanically ventilated patients: validity and reliability of the confusion assessment method for the intensive care unit (CAM-ICU). JAMA 2001; 286: 2703-10.

15 Reade MC, Eastwood GM, Bellomo R, et al. Effect of dexmedetomidine added to standard care on ventilator-free time in patients with agitated delirium: a randomized clinical trial. JAMA 2016; 315: 1460-8.

16 Shehabi Y, Howe BD, Bellomo R, et al. Early sedation with dexmedetomidine in critically ill patients. N Engl J Med 2019; 380: 2506-17.

17 Devlin JW, Roberts RJ, Fong JJ, et al. Efficacy and safety of quetiapine in critically ill patients with delirium: a prospective, multicenter, randomized, double-blind, placebo-controlled pilot study. Crit Care Med 2010; 38: 419-27.

18 Pandharipande PP, Ely EW, Arora RC, et al. The intensive care delirium research agenda: a multinational, interprofessional perspective. Intensive Care Med 2017; 43: 1329-39.

19 Perez D, Peters K, Wilkes $L$, et al. Physical restraints in intensive care - an integrative review. Aust Crit Care 2019; 32: 165-74.

20 Pol A, Carter M, Bouchoucha S. Violence and aggression in the intensive care unit: what is the impact of Australian National Emergency Access Target? Aust Crit Care 2019; 32: 502-8. 